\title{
Recent Studies of Little Higgs Models in ATLAS
}

\author{
Fabienne Ledroit \\ LPSC; 53 avenue des Martyrs; F-38026 Grenoble CEDEX
}

\begin{abstract}
The ATLAS Collaboration at the LHC continues investigating the possibility to detect particles predicted by Little Higgs models. In this talk, the latest results on the $Z / W h$ decays and on the hadronic decays of the new gauge bosons $Z_{H} / W_{H}$ are reviewed.
\end{abstract}

Keywords: Little Higgs, LHC, heavy gauge bosons

PACS: $12.38 . \mathrm{Qk}, 12.60 . \mathrm{Cn}, 14.70 . \mathrm{Pw}$

\section{INTRODUCTION}

The Higgs sector of the Standard Model (SM) contains an instability due to the radiative corrections to the Higgs boson mass; this is known as the hierarchy problem. Various theories and models have been designed to solve this problem. One of them is called the Little Higgs model and was proposed a few years ago [1]. This is an effective model in which the Higgs is a pseudo-Goldstone boson resulting from a spontaneously broken global symmetry at a very high scale. Such a Higgs boson remains light, protected by the approximate global symmetry. Here we concentrate on one of the several implementations of the model, called the Littlest Higgs model [2]. This particular model includes a $S U(5)$ to $S O(5)$ breaking, and the following heavy new particles: scalar particles $\phi^{0}$, $\phi^{+}, \phi^{++}$, new $2 / 3$ charge heavy quark $(T)$, and heavy partners of the electroweak gauge bosons $A_{H}, Z_{H}$ and $W_{H}^{ \pm}$.

\section{PHENOMENOLOGY AT THE LHC}

The Higgs boson $(h)$ properties remain those of the SM. Production cross sections and branching ratios of the heavy new particles have been computed in [3, 4], leading to the following search strategy:

- The $\phi^{++}$scalar is produced in $W^{+} W^{-}$fusion. The dominant decay mode is $\phi^{++} \rightarrow$ $W^{+} W^{-}$. The corresponding cross section is small and the SM background large, so this particle would be difficult to observe. Nevertheless, the prospects for its detection have been studied and reported in [5].

- The heavy quark $T$ can be produced singly in the $b q \rightarrow T q^{\prime}$ channel via $W$ exchange. The $T$ then decays to $t Z$ or $t h$. The corresponding cross section is small as well, although larger than the cross section for the QCD production of a $T \bar{T}$ pair. How well the $T$ could be detected by ATLAS was studied in [5].

- The gauge bosons $Z_{H}$ and $W_{H}$ are produced via $q \bar{q}^{(\prime)}$ annihilation and the cross section is much higher than for the $\phi^{++}$and $T$. Once their mass is fixed (they are 
approximatly degenerate), the only parameter is $\theta$, the mixing angle between the $W$ triplets, and the cross section is proportional to $(\cot \theta)^{2}$. The production cross section for the $A_{H}$ boson is more difficult to predict since the couplings are not entirely fixed by the model. Nevertheless, some results on its search are shown in [5].

In the following, we concentrate on the experimental search of $Z_{H}$ and $W_{H}$ using the ATLAS detector. The PYTHIA event generator[6] was used to generate signal and background events. The events were passed through the ATLAS fast simulation[7] which provides a parameterized response of the detector to jets, electrons, muons, isolated photons and missing transverse energy. The integrated luminosity is assumed to be $300 \mathrm{fb}^{-1}$, which corresponds to data collected during three years of running at high luminosity. The event selection includes the ATLAS trigger criteria.

\section{HEAVY GAUGE BOSON SEARCHES}

Once produced, the heavy $Z_{H}$ and $W_{H}$ bosons can have two types of decays: the fermionic channels, which are the same channels as their SM partners, and the bosonic channels. Denoting $V_{H}$ for both $Z_{H}$ and $W_{H}$ (and $V$ for $Z$ and $W$ ), these are $V_{H} \rightarrow V h$ and $Z_{H} \rightarrow W^{+} W^{-}, W_{H} \rightarrow Z W$.

Among the fermionic channels, $Z_{H} \rightarrow l^{+} l^{-}$and $\mathrm{W}_{H} \rightarrow l v$ are clearly the discovery channels. It was shown in [5] that $V_{H}$ can be discovered at masses of up to $6 \mathrm{TeV}$ if $\cot \theta$ is large. On the other hand, the $V_{H} \rightarrow q \bar{q}^{(\prime)}$ channel is expected to be more difficult, because of the high level of background. It was studied however, and the results are reported below.

The observation of the $V_{H} \rightarrow V h$ decay is essential to test Little Higgs models. Besides, it is dominant at low values of $\cot \theta$. A first study was performed assuming that the Higgs boson is discovered with a mass $m_{h}=120 \mathrm{GeV}$ [5]. The resulting exclusion contour in the $\left(M_{V_{H}}, \cot \theta\right)$ plane is shown on Fig 1 (left). In the next subsection, we report on the results of a more recent study of this decay, which assumes $m_{h}=200 \mathrm{GeV}$.

\section{Search for the decays $V_{H} \rightarrow V h$ assuming $m_{h}=200 \mathrm{GeV}$.}

When $m_{h}=200 \mathrm{GeV}$, the main Higgs decays are $h \rightarrow W^{+} W^{-}(73 \%)$ and $h \rightarrow Z Z$ (26\%). Various $Z h$ and $W h$ final states have been chosen, as listed in Table 1 resulting from a compromise between cross section and signature [8]. In all the modes, the main background is inclusive top pair production, where both tops decay semi-leptonically and a third lepton can arise from a $b$ jet. In the A1 and A2 modes, the missing transverse momentum is used to reconstruct the Higgs momentum, assuming that the neutrino is colinear to the leptons. In addition, the $W$ mass constraint is applied in the B1 and B3 mode. The A3 and A4 modes have indistinguishable final state. The hadronic decay of high $p_{T} W$ or $Z$ are reconstructed by looking for two high $p_{T}$ jets with mass close to the $W / Z$ mass or, if it fails, by taking the jet with the largest $p_{T}$. 

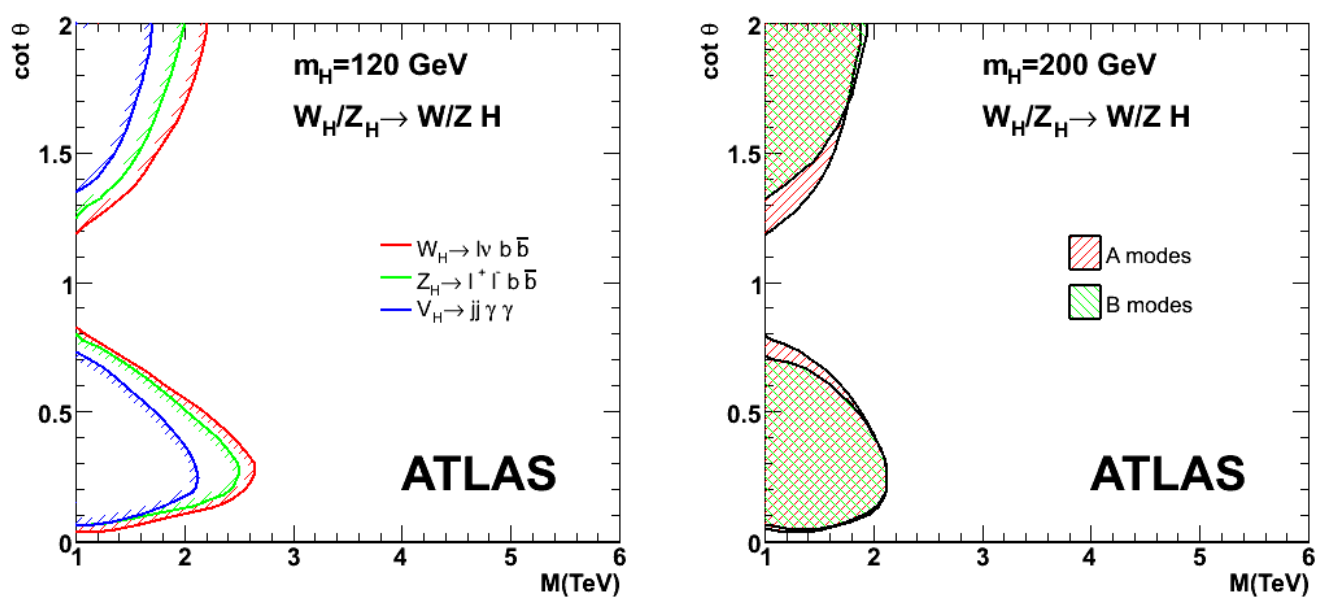

FIGURE 1. Discovery region with the $V_{H} \rightarrow V h$ channel (left: $m_{h}=120 \mathrm{GeV}$, right: $m_{h}=200 \mathrm{GeV}$ ) as a function of $M_{V_{H}}$ and $\cot \theta$.

TABLE 1. $Z_{H}$ and $W_{H}$ final states under study. The branching ratios are computed assuming $\cot \theta=0.5$, the selection efficiencies $\varepsilon$ assume a gauge boson mass of $1 \mathrm{TeV}$.

\begin{tabular}{cclll}
\hline Mode & BR $\left(10^{-4}\right)$ & \multicolumn{1}{c}{ Decay } & Signature & $\varepsilon$ \\
\hline A1 & 1.0 & $Z_{H} \rightarrow Z h \rightarrow l^{+} l^{-} W^{+} W^{-} \rightarrow l^{+} l^{-} l^{+} v l^{-} v$ & 4 leptons $+E_{T}^{\text {miss }}$ & $34 \%$ \\
A2 & 3.0 & $W_{H} \rightarrow W h \rightarrow l v-W^{+} W^{-} \rightarrow l v l^{+} v l^{-} v$ & 3 leptons $+E_{T}^{\text {miss }}$ & $12 \%$ \\
A3 & 0.4 & $Z_{H} \rightarrow Z h \rightarrow j j Z Z \rightarrow j j l^{+} l^{-} l^{+} l^{-}$ & 4 leptons + jets & $26 \%$ \\
A4 & 0.4 & $W_{H} \rightarrow W h \rightarrow j j Z Z \rightarrow j j l^{+} l^{-} l^{+} l^{-}$ & 4 leptons + jets & \\
B1 & 6.8 & $Z_{H} \rightarrow Z h \rightarrow l^{+} l^{-} W^{+} W^{-} \rightarrow l^{+} l^{-} j j l v$ & 3 leptons + jets $+E_{T}^{\text {miss }}$ & $22 \%$ \\
B2 & 0.8 & $Z_{H} \rightarrow Z h \rightarrow l^{+} l^{-} Z Z \rightarrow l^{+} l^{-} j j l^{+} l^{-}$ & 4 leptons + jets & $17 \%$ \\
B3 & 2.4 & $W_{H} \rightarrow W h \rightarrow l v Z Z \rightarrow l v j j l^{+} l^{-}$ & 3 leptons + jets & $15 \%$ \\
\hline
\end{tabular}

The corresponding discovery reach is summarised on Fig 1 (right); it is similar to the $m_{h}=120 \mathrm{GeV}$ case, but the bound on the $V_{H}$ mass is weaker ( $6 \mathrm{TeV}$ versus $2 \mathrm{TeV}$ ).

\section{Search for hadronic decays}

For $\cot \theta \approx 1, \mathrm{BR}\left(V_{H} \rightarrow V h\right)$ vanishes and the branching ratios to heavy quarks are [4]: $\mathrm{BR}\left(Z_{H} \rightarrow b \bar{b}\right)=\mathrm{BR}\left(Z_{H} \rightarrow t \bar{t}\right)=1 / 8$ and $\mathrm{BR}\left(W_{H} \rightarrow t b\right)=1 / 4$. It was shown[9] that no convincing signal can be seen in the $Z_{H}$ case. However, the $W_{H} \rightarrow t b$ appears visible in the channel where the top decays to the $W(l v) b$ final state. One isolated lepton is searched for, and two $b$-jets tagged, one close to the lepton, one recoiling against the lepton. The neutrino 3-momentum is estimated from the reconstructed missing transverse momentum and assuming it is parallel to the lepton momentum.

The discovery reach is shown on Fig 2 in the same plane as before, together with earlier results on the $Z_{H} \rightarrow l^{+} l^{-}$and $W_{H} \rightarrow l v$ discovery channels [5]. The $\cot \theta=1$ region which was missing in the $V_{H} \rightarrow V h$ analyses is well covered up to $M_{W_{H}}=2.5 \mathrm{TeV}$. 

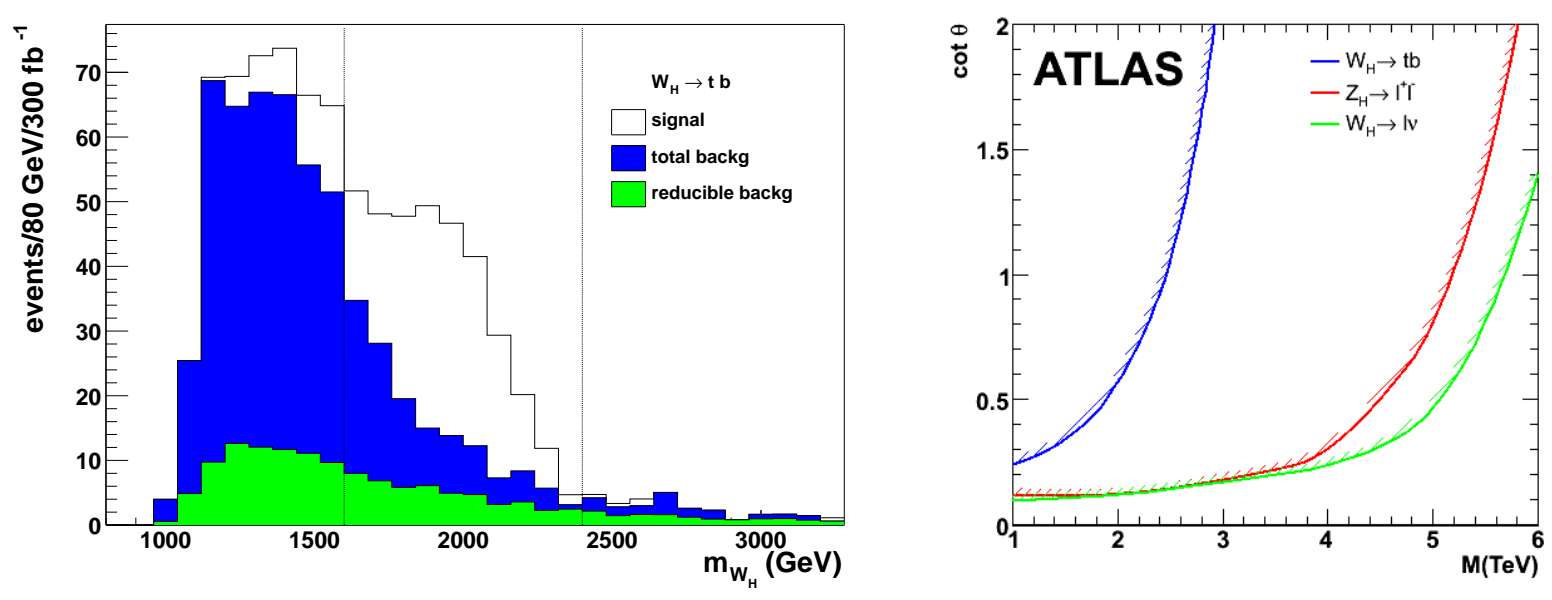

FIGURE 2. Left: reconstructed mass peak for $W_{H} \rightarrow t b\left(M_{W_{H}}=2 \mathrm{TeV}\right)$. Right: discovery region with the fermionic channels as a function of $M_{V_{H}}$ and $\cot \theta$.

\section{SUMMARY}

We have shown the latest examples of channels which can be used to test the predictions of Little Higgs models. The discovery reach is found to be rather good, in particular for the new gauge bosons. However, we are aware that the model family of the particular implementation studied here is actually disfavoured by precision electroweak constraints. The introduction of a discrete symmetry called T-parity would allow to escape them. Unfortunately, since it implies that the heavy partners can only be produced in pairs, the phenomenology is drastically modified and would therefore require a new analysis.

\section{ACKNOWLEDGMENTS}

Thanks are due to the authors of these analyses: J.E. Garcia, S. González de la Hoz, M. Lechowski, L. March, E. Ros and, last but not least, D. Rousseau.

\section{REFERENCES}

1. N. Arkani-Hamed, A. G. Cohen, and H. Georgi, Phys. Lett. B 513, 232 (2001).

2. N. Arkani-Hamed, A. G. Cohen, E. Katz, and A. Nelson, JHEP 0207, 034 (2002).

3. G. Burdman, M. Perelstein and A. Pierce, Phys. Rev. Lett. 90, 241802 (2003) [Erratum-ibid. 92, 049903 (2004)]

4. T. Han, H. E. Logan, B. McElrath, and L. T. Wang, Phys. Rev. D 67, 095004 (2003).

5. G. Azuelos et al., Eur. Phys. J C39S2, 13-24 (2005).

6. T. Sjöstrand et al., Comput. Phys. Commun. 135, 238 (2001).

7. D. Froidevaux, E. Richter-Waş, and L. Poggioli, ATLFAST 2.0: a fast simulation package for ATLAS, ATL-PHYS-98-131 (1998).

8. E. Ros, and D. Rousseau, Little Higgs studies in ATLAS, ATL-PHYS-CONF-2006-007 (2006).

9. S. González de la Hoz, L. March, and E. Ros, Search for hadronic decays of $Z_{H}$ and $W_{H}$ in the Little Higgs model, ATL-PHYS-PUB-2006-003 (2006). 